\title{
Amorphous clusters in Co implanted ZnO induced by boron pre-implantation
}

K. Potzger, A. Shalimov, Shengqiang Zhou, H. Schmidt, A. Mücklich, M. Helm, and J. Fassbender,

Institut für lonenstrahlphysik und Materialforschung, Forschungszentrum DresdenRossendorf e.V., Bautzner Landstraße 400, 01328 Dresden, Germany

M. Liberati and E. Arenholz

Advanced Light Source, Lawrence Berkeley National Laboratory, Berkeley, CA 94720, USA

We demonstrate the formation of superparamagnetic/ferromagnetic regions within $\mathrm{ZnO}(0001)$ single crystals sequently implanted with $\mathrm{B}$ and $\mathrm{Co}$. While the preimplantation with B plays a minor role for the electrical transport properties, its presence leads to the formation of amorphous phases. Moreover, B acts strongly reducing on the implanted Co. Thus, the origin of the ferromagnetic ordering in local clusters with large Co concentration is itinerant d-electrons as in the case of metallic Co. The metallic amorphous phases are non-detectable by common X-ray diffraction. 


\section{Introduction}

Ferromagnetic diluted magnetic semiconductors (DMS) are currently intensely studied due to their potential applications in spin electronics. Among them, Co doped $\mathrm{ZnO}$ has been widely characterized with respect to its magnetic properties. Those range from paramagnetic for perfect crystalline structure $^{1}$ to ferromagnetic/ superparamagnetic if secondary inclusions ${ }^{2}$ or defects ${ }^{3}$ are present. Experimentally, two kinds of crystalline secondary inclusions are observed, either compounds involving transition metal (TM) ions or metallic TM clusters. If such phases are avoided, one possibility to create a ferromagnetic DMS from Co doped $\mathrm{ZnO}$ is considered to be the enhancement of n-type conductivity in order to boost Zenerexchange interaction between the localized moments of the $\mathrm{Co}^{2+}$ ions. For that purpose $\mathrm{ZnO}$ is often doped with a group III element like $\mathrm{B}^{4}, \mathrm{Al}$ or $\mathrm{Ga}$. To evaluate the origin of the ferromagnetic properties, however, one has to consider that those dopants not only have influence of the electronic but also on the structural properties of the host material. Demonstrating those effects, we investigated the case of significant boron co-doping. Boron is well known to amorphize metals like Fe, Co or their alloys. Moreover, in Ref. 5 it is suggested that boron impurities in $\mathrm{ZnO}$ might form complexes with oxygen interstitials and stabilize oxygen vacancies, which in turn favours the vacancy agglomeration process. Thus, the following effects are expected from the presence of boron

1. Creation of stable amorphous phases

2. Reduction of Co to metallic state With respect to amorphization, we expect similar results as found by Kucheyev et al. ${ }^{11}$ who implanted $\mathrm{Si}$ ions into $\mathrm{ZnO}$ single crystals and observed a disordered layer mainly in the rising edge of the implantation depth profile. The layer contained a mixture of nanoscale amorphous and embedded crystalline regions. Amorphous 
phases in TM doped $\mathrm{ZnO}$ have been observed also without co-doping. In a recent paper $^{6}$ we showed that for Fe implanted $\mathrm{ZnO}$ single crystals, metallic crystalline $\mathrm{Fe}$ clusters are formed during low temperature vacuum annealing. With increasing temperature, the $\mathrm{ZnO}$ matrix locally starts to decompose and evidence for small amorphous Fe-Zn-O complexes was found. Those amorphous inclusions are nonmagnetic. Note that the tendency of oxidizing Fe, i.e., the formation of such amorphous non-magnetic complexes is larger for $\mathrm{ZnO}$ of low crystalline quality, i.e. such containing large amounts of microscopic defects like grain boundaries. In such crystals, formation of crystalline secondary phases is suppressed ${ }^{7}$. Note that the expression "low crystalline quality" indicating large amounts of defects, mosaicity and grain boundaries should not be confused with "low crystallinity" or "amorphous" in the sense of low or absent long range crystalline order. Upon long time annealing, the non-magnetic complexes turn into crystalline inverse spinel $\mathrm{ZnFe}_{2} \mathrm{O}_{4}$ clusters which are ferrimagnetic. If the crystalline clusters are very small and occur in low density, they are difficult to detect by common structural analysis methods. Nevertheless, high resolution and sensitivity methods like synchrotron radiation x-ray diffraction (XRD) allow the detection of such clusters ${ }^{8}$. In contrast to that, amorphous or low crystalline inclusions are not detectable by the usual XRD methods. Unlike Fe, metallic crystalline Co cluster formation cannot be suppressed by lowering the crystalline quality. Instead, tetragonal coordination of $\mathrm{Co}^{2+}$ ions in the $\mathrm{ZnO}$ matrix or metallic precipitates appear to be favoured upon moderate-fluence and low-temperature $\mathrm{Co}^{+}$ implantation ${ }^{9}$. Large fluence implantation of $\mathrm{Co}^{+}$, on the other hand, leads to the formation of superparamagnetic, structurally disordered Co-Zn-O clusters ${ }^{10}$. The saturation magnetisation per implanted Co ion increases with increasing $\mathrm{Co}^{+}$fluence. Those clusters are also undetectable by $\mathrm{XRD}$, despite the rather large saturation magnetization of the samples. 


\section{Experiment}

We used hydrothermally grown $\mathrm{ZnO}(0001)$ single crystals polished on both dsides provided by CRYSTEC (Berlin, Germany). We implanted $\mathrm{B}^{+}$ions at an energy of 30 $\mathrm{keV}$, an angle of $7^{\circ}$, and different fluences summarized in Table I. The sample holder was cooled by liquid nitrogen during implantation. The $\mathrm{Co}^{+}$ions were implanted subsequently using the same angle and cooling, but at an energy of $80 \mathrm{keV}$ as in Ref. 5. The majority of analytic techniques have been applied to samples implanted with $\mathrm{Co}^{+}$at a fluence of $8 \times 10^{16} \mathrm{~cm}^{-2}$. For comparison, three samples were implanted with boron at different fluences and $16 \times 10^{16} \mathrm{Co}^{+} / \mathrm{cm}^{2}$ (Table I).

Table I: Identifiers of the $\mathrm{ZnO}(0001)$ crystals and implanted ion fluences. All implantations took place at an angle of $7^{\circ}$ and with samples mounted at a liquidnitrogen-cooled target.

\begin{tabular}{|l|l|l|}
\hline Identifier & $\begin{array}{l}\mathrm{B}^{+} \text {fluence } \\
\left(\mathrm{cm}^{-2}\right)\end{array}$ & $\begin{array}{l}\mathrm{Co}^{+} \text {fluence } \\
\left(\mathrm{cm}^{-2}\right)\end{array}$ \\
\hline$Z(0,8)$ & - & $8 \times 10^{16}$ \\
\hline$Z(0.5,8)$ & $5 \times 10^{15}$ & $8 \times 10^{16}$ \\
\hline$Z(2,8)$ & $2 \times 10^{16}$ & $8 \times 10^{16}$ \\
\hline$Z(4,8)$ & $4 \times 10^{16}$ & $8 \times 10^{16}$ \\
\hline$Z(8,8)$ & $8 \times 10^{16}$ & $8 \times 10^{16}$ \\
\hline$Z(16,8)$ & $1.6 \times 10^{17}$ & $8 \times 10^{16}$ \\
\hline$Z(32,8)$ & $3.2 \times 10^{17}$ & $8 \times 10^{16}$ \\
\hline
\end{tabular}

\begin{tabular}{|l|l|l|}
\hline$Z(0,16)$ & - & $16 \times 10^{16}$ \\
\hline
\end{tabular}




\begin{tabular}{|l|l|l|}
\hline$Z(8,16)$ & $8 \times 10^{16}$ & $16 \times 10^{16}$ \\
\hline$Z(32,16)$ & $32 \times 10^{16}$ & $16 \times 10^{16}$ \\
\hline
\end{tabular}

For the fluence of $8 \times 10^{16} \mathrm{Co}^{+} / \mathrm{cm}^{2}$, the implantation energies were chosen in such way that the maximum of the Co depth profile overlaps with the rising edge of the $\mathrm{B}^{+}$ depth profile calculated using the TRIDYN $\operatorname{code}^{12}$ (Fig. 1).

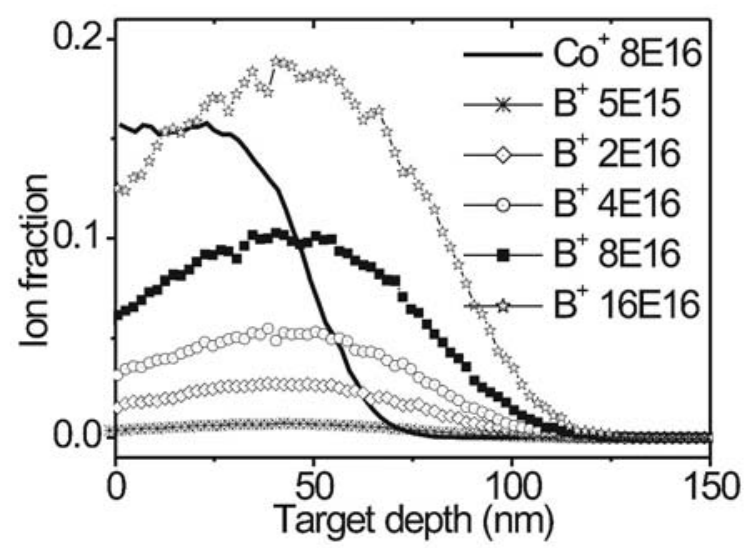

Fig. 1. TRIDYN simulation displaying the expected depth profiles of the implanted ions. Sputtering was taken into account.

Sample characterization was performed by superconducting quantum interference device (SQUID) magnetometry using a Quantum Design MPM S XL, atomic force microscopy (AFM) using a Veeco/DI Multimode, x-ray diffraction using a Siemens D5005 diffractometer, transmission electron microscopy (TEM) using a FEI Titan and magneto-transport using the van der Pauw geometry. $X$ ray absorption (XA) spectra were measured at the Advanced Light Source at beamline 4.0.2 in total electron yield (TEY) mode by monitoring the sample drain current thus probing the surface near region of the samples. $\mathrm{XMCD}$ spectra were acquired at $15 \mathrm{~K}$ in a magnetic field of 0.55T applied collinear with the x-ray beam which in turn impinged on the sample surface at and angle of $30^{\circ}$ to the sample surface. 


\section{Structural properties}

The behaviour of the $\mathrm{ZnO}$ surface region was evaluated by $\mathrm{AFM}^{12}$. For samples without implantation, $\mathrm{R}_{\mathrm{q}}$ amounts to approximately $0.2 \mathrm{~nm}$ but surface roughening can be observed for all samples listed in Table I after implantation. No dependence of the surface RMS roughness $\left(R_{q}\right)$ on the $B^{+}$fluence $\left(\Phi_{B}\right)$ was found. Rather, $R_{q}$ oscillates between a maximum value of $3.7 \mathrm{~nm}$ and a minimum value of $0.5 \mathrm{~nm}$ (not shown). In contrast, all implanted samples show regular meander like structures. Figs. 2(a)-(c) display AFM micrographs for selected B-containing samples.
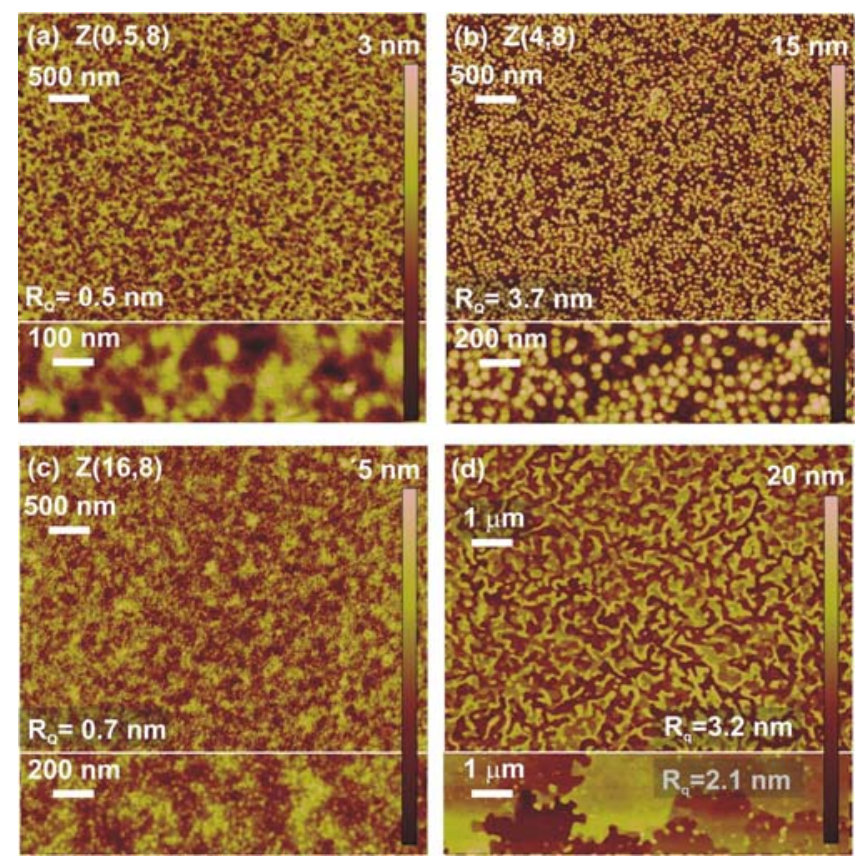

Fig. 2(a)-(c). Surface topography after implantation. The sample identifiers and RMS roughness $R_{q}$ are indicated. Note that the implantation took place into the $\mathrm{Zn}$ face. (d). AFM topography of $\mathrm{ZnO}$ single crystals annealed at $1313 \mathrm{~K}$ in high vacuum $\left(2.5 \times 10^{-6} \mathrm{mbar}\right)$ for $15 \mathrm{~min}$ with $\mathrm{Zn}$ face up. The inset shows the corresponding $\mathrm{O}$ face annealed sample.

The only systematic trend is that for low fluences, i.e. $Z(0 \ldots 2,8)$, the meanders do not exhibit an inner structure. Exemplarily, the surface topography for $Z(0.5,8)$ is shown 
in Fig. 2(a) (inset with larger magnetification). In contrast, for samples $Z(4 \ldots 32,8)$ the meanders consist of nanoscale clusters. For $Z(4,8)$ in Fig. 2(b) they are nearly non dispersed and exhibit $50 \mathrm{~nm}$ diameter and protrude about $10 \mathrm{~nm}$. Note that the micrographs are not fully representative for the whole sample surface. Instead, there are roughness variations which are most pronounced for samples $Z(0,8)$ and $Z(8,8)$. Note that the ion implantation occurred under high vacuum conditions. As stated in Ref. 9, the formation of meander like hillocks results from the steady state of $\mathrm{ZnO}$ decomposition and thus $\mathrm{Zn}$ and $\mathrm{O}$ evaporation from the surface ${ }^{14}$ on one hand and re-growth on the other hand. Self organisation from ion beam erosion, which is caused by a thin amorphous layer that forms during the very first seconds of the sputtering process ${ }^{15}$, can be excluded. This becomes clear from Fig. 2(d) where $\mathrm{ZnO}$ single crystals were annealed in vacuum without any implantation. Similarities between the annealed and the implanted samples (Fig. 2(a)-(c)), i.e. meander like self-organized regular structures, are immediately evident for the $\mathrm{Zn}$-face. The annealed O-face (inset in Fig. 2(d)) exhibits lower roughness and different features. Note, however, that the time for the Co implantation scaled with the implanted B fluence with a maximum of 7 hours, i.e., being much larger than the annealing time. Phenomena known from self organization or chaos theory like self similarity or fractal dimensions (inset in Fig. 2(d)) are evident. Ion beam erosion induced self organisation might be responsible for the inner structure of the meanders, i.e. nanoscale dots. At low energy/high fluence implantation, such dots can be observed in various materials ${ }^{15}$. We would like to conclude that the preparation has the following two effects on the surface relevant for the magnetic properties:

- Formation of meanders of variable size

- Formation of nanoclusters 
Such behaviour is expected for the entire near surface region. The TEM micrograph of the sample $Z(0,8)$ implanted only with $8 \times 10^{16} \mathrm{Co}^{+} / \mathrm{cm}^{2}$ shows mainly a crystalline Wurtzite structure with defective regions induced by the ion bombardment (inset in Fig. 3(a)). As in Ref. 10, possible near-surface amorphous or low-crystalline regions cannot be detected since the background Wurtzite lattice dominates the TEM micrograph (not shown). In contrast, for samples $Z(8,8)$ and $Z(32,8)$, which have been investigated exemplarily by TEM, a large structural disorder including low crystalline/amorphous regions as well as secondary phases can be observed. Fig. 3 shows interface (a),(c) and surface (b),(d) near regions as well as the surface near region in lower magnetification (insets) of both samples. 


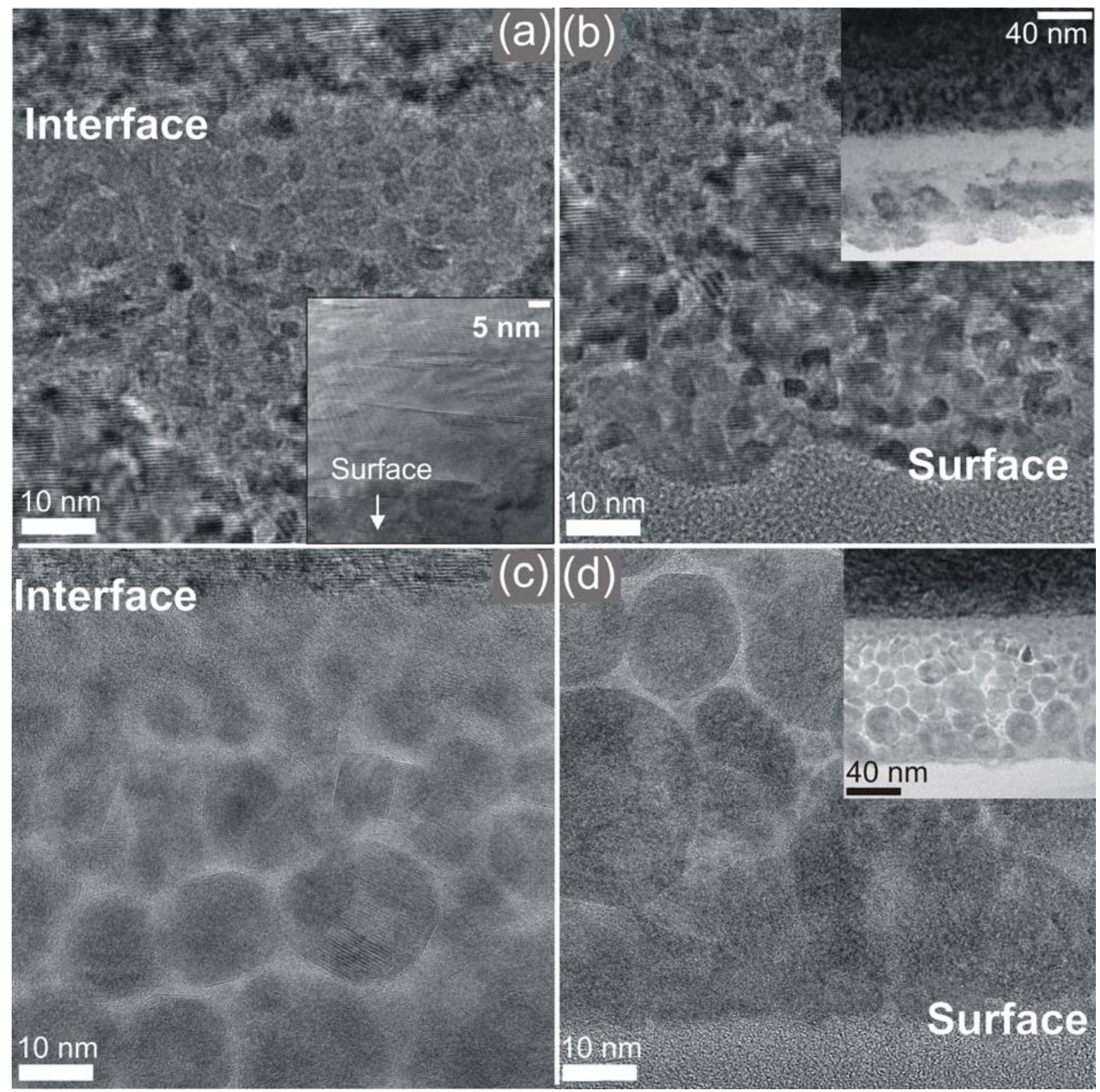

Fig. 3 Surface and interface near regions investigated by TEM for $Z(8,8)(a)$, (b) and $Z(32,8)$ (c) (d). The inset in (a) shows the near surface region of sample $Z(0,8)$. The insets in (b) and (d) show the surface near region with lower magnification illustrating the predominantly amorphous (bright) and long range ordered (dark) area.

From the TEM results it is evident that the thickness of the predominantly amorphous (bright) film increases with increasing boron fluence from $80 \mathrm{~nm}$ for $Z(8,8)$ to $120 \mathrm{~nm}$ for $Z(32,8)$, respectively. This is expected from the amorphization stimulated chemically by the boron. For $Z(8,8)$, no clear conclusions can be drawn on the 
(crystalline) inclusions. In contrast, for $\mathrm{Z}(32,8)$ it can be found by Fast Fourier transform (FFT) that the $\sim 5 \mathrm{~nm}$ thick layer at the surface consists of small clusters with $\mathrm{ZnO}$ lattice constant. Below that layer there is a large amorphous/low crystalline area with some crystalline $\mathrm{Zn}(\mathrm{Co}) \mathrm{Co}_{2} \mathrm{O}_{4}$, $\mathrm{CoO}$ as well as metallic Co inclusions. Those are too few to be detected by XRD. The layer above the interface with the long range ordered $\mathrm{ZnO}$ bulk is rather thin $(\sim 5-10 \mathrm{~nm})$ and amorphous. The amorphous clusters in the middle region show bright cores (Fig. 3(c)) which do not contain Co as found by EFTEM (Fig. 4). Thus, the Co density strongly varies within the $\sim 20 \mathrm{~nm}$ sized Co-Zn-O clusters. At some spots the Co density becomes very large without creation of a long range ordered crystalline phase (Fig. 4(b)). Those are responsible for the magnetic moment observed.

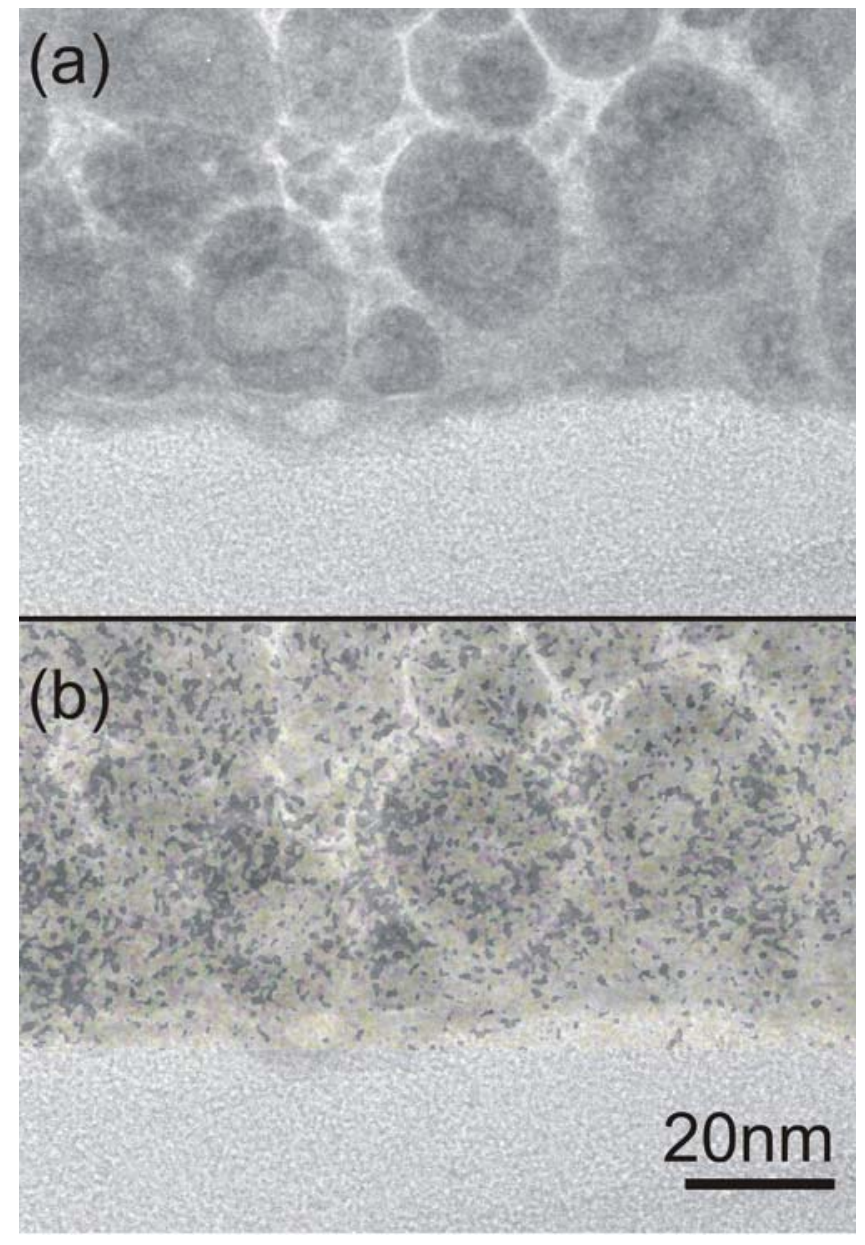

Fig. 4 (a),(b). TEM micrographs of the same region measured for sample $Z(32,8)$. (b) contains an overlay with the EFTEM image measured at the Co emission edge. Gray 
spots correspond to large Co concentration. Note that the contrast of the overlay has been artificially enhanced for better visibility and must be considered as an illustration rather than an actual Co map.

\section{Magnetometry}

Directly after implantation, the samples have been analysed by magnetometry. We performed both magnetization (M) vs. temperature $(T)$ as well as $M$ vs. field $(H)$ measurements. The M-T curves have been obtained using the zero-fieldcooling/field-cooling (ZFC/FC) protocol at a constant field of $100 \mathrm{Oe}$. The M-H curves are recorded at $5 \mathrm{~K}$ and $300 \mathrm{~K}$. Exemplarily, sample $\mathrm{Z}(4,8)$ was investigated with respect to large field effects and anisotropy (both not shown). It was found that the M$\mathrm{H}$ dependency at $5 \mathrm{~K}$ consists of a part dominated by ferro-/superparamagnetism in the range of approximately $\pm 5 \mathrm{kOe}$. The background in this range can be approximated by a line. Above (below) those values, a well separated part dominated by paramagnetism in the measured range up to $65 \mathrm{kOe}$ was observed. Moreover, the sample had a clear hard magnetization direction along the (0001) axis and easy magnetization directions in plane. This was confirmed also using sample $Z(8,8)$. For our measurements thus we have chosen a field range between $\pm 5 \mathrm{kOe}$ and we measured along the in-plane easy-axis direction, where the hysteresis loops are fully saturated. Figures. 5(a)-(d) show ZFC/FC M-T and M-H curves for selected samples. For increasing $\mathrm{B}^{+}$fluence, the maxima in the ZFC curve shift above $300 \mathrm{~K}$ and the saturation magnetization $\mathrm{M}_{\text {sat }}$ rises by a factor of 10 at its maximum. This behaviour is reflected by Fig. $6(a)$. With increasing $B^{+}$fluences $M_{\text {sat }}$ decreases again. Note that the maximum occurs at lower $\mathrm{B}^{+}$fluences for loops measured at $300 \mathrm{~K}$. Qualitatively, the curves from Figs. 5 and 6(a) can be interpreted as follows: One general feature of superparamagnetism is the drastic drop of $\mathrm{M}_{\text {sat }}$ and $\mathrm{H}_{\mathrm{C}}$ with increasing temperature 
(exponential decay). Another feature is the increase of $\mathrm{H}_{\mathrm{C}}$ with cluster size and interparticle interaction. These characteristics are different from ferromagnetic material where $M_{\text {sat }}-T$ is described by the Curie law. The drastic drop of $H_{c}$ with temperature increase is observed in all $\mathrm{M}-\mathrm{H}$ curves shown in Fig. 5 indicting that all samples are largely superparamagnetic. $Z(4,8)$ shows largest $H_{C}$ and lowest drop of $M_{\text {sat }}$ with temperature (Fig. 5(c)). Thus, although it does not exhibit the largest $M_{\text {sat }}$, it is the most "ferromagnetic" sample. The strongest superparamagnets are $Z(0.5,8)$ in Fig. 5(a), $Z(2,8)$ (not shown), and $Z(32,8)$ in Fig. 5(d). Here the drop of $M_{\text {sat }}$ with increasing temperature is largest. In Fig. 5(a) also a "wasp-waist" hysteresis can be observed which often occurs in implanted oxides containing small superparamagnetic clusters. 

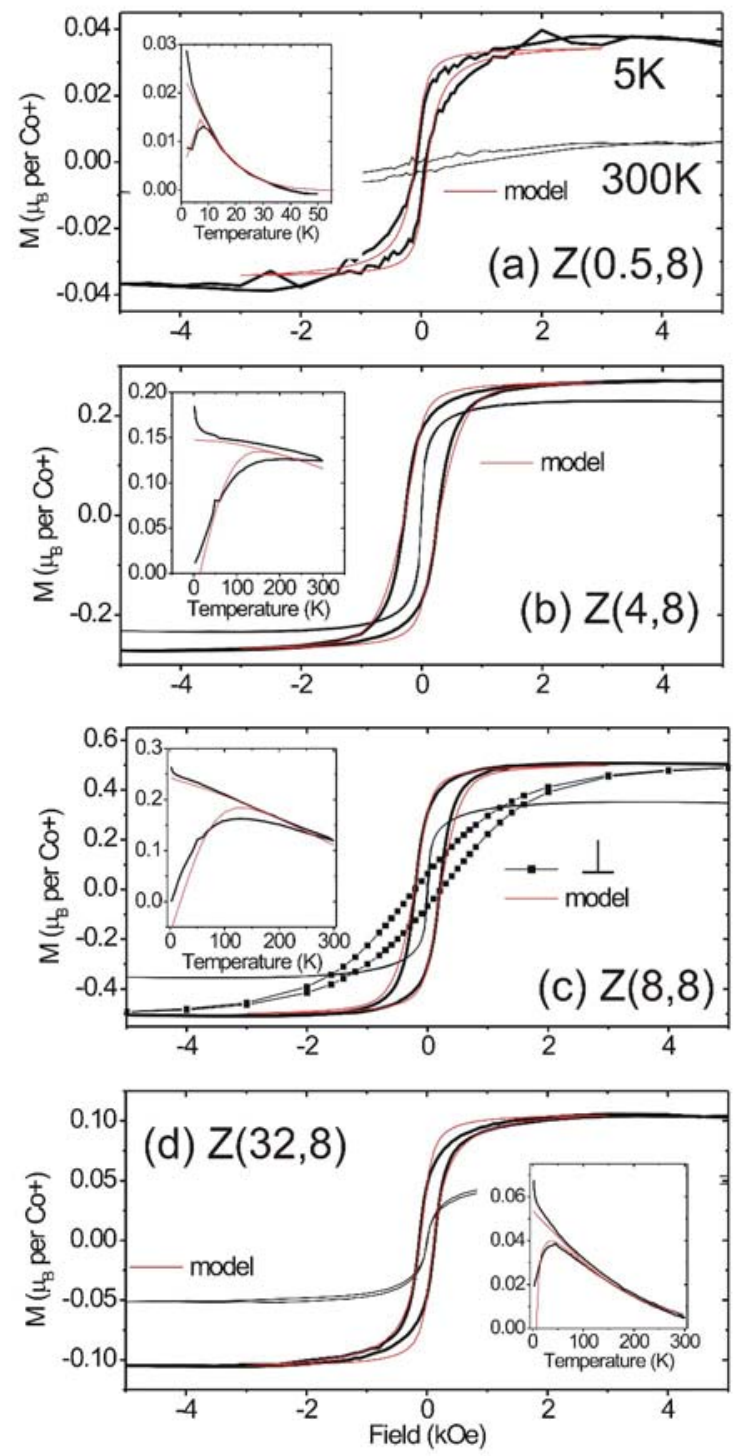

Fig. 5. Magnetization (M) vs. field and temperature (insets) curves for selected samples. The fluences are indicated using the notation introduced in Table I. Note that the ZFC curves except for $\mathrm{Z}(0.5,8)$ exhibit a small feature around $50 \mathrm{~K}$. It is related to residual molecular oxygen at the sample and is thus a typical artefact of the SQUID measurement.

Quantitatively, the curves displayed in Fig. 5 can be modelled using the Preisach approach introduced by $\mathrm{T}$. Song et $\mathrm{al}^{16}{ }^{16}$. The temperature dependence of the parameter $p$ describing the magnetic properties of the (nano)phase is usually 
expressed by the critical temperature $T_{C}$ and the critical exponent $\Gamma$ : $p=p_{0}\left(1-\frac{T}{T_{C}}\right)^{\Gamma}$, with $p$ substituted by the mean magnetic moment $\mu$ of an individual cluster, the mean coercivity $H_{c}$, or the dispersions $\sigma_{i}$ and $\sigma_{c}$ of short range interparticle interaction and coercive field. Among the boron pre-implanted samples, the parameters $\sigma_{\mathrm{c} 0}$ and $\sigma_{\mathrm{i} 0}$ (not shown) exhibit a maximum for sample $Z(4,8)$. As discussed qualitatively, $Z(4,8)$ shows strongest interparticle interaction leading to "most" ferromagnetic behaviour. The critical temperature $T_{C}$ (not shown) rises from $60 \mathrm{~K}$ for $\mathrm{Z}(0.5,8)$ to $240 \mathrm{~K}$ for $\mathrm{Z}(2,8)$ up to $600 \mathrm{~K}$ for $\mathrm{Z}(4 \ldots 36,8)$. Fig. $6(\mathrm{~b})$ displays the evolution of $\mu_{0}$ and $N$, i.e. the number of clusters per $\mathrm{cm}^{2}$ implanted. The most striking feature is the difference between both parameters for samples $Z(8,8)$ and $Z(16,8)$. Although both exhibit maximum $M_{\text {sat }}$ (Fig. 6(a)), the former is characterised by maximum $\mu_{0}$ and the latter by maximum $N$. Note that the saturation magnetization of $Z(0,8)$ and $Z(0.5,8)$ are similar. There is, however, a strong difference between the coercivities (Fig. 6(a)) and the $\sigma_{\mathrm{C} 0}$ values (Fig. 6(b)). Generally, $\mathrm{H}_{\mathrm{C} 0}$ had to be set to zero for all samples containing boron while its non-zero for $Z(0,8)^{9}$. Comparing a boron pre-implanted with a non pre-implanted sample, e.g. $Z(0,8)$ with $Z(0.5,8)$ it becomes evident that for the former the coercivity $\mathrm{H}_{\mathrm{C}}$ as well as the dispersions $\sigma_{\mathrm{co}}$ and $\sigma_{i 0}$ (latter not shown) are significantly larger as for the latter. Although the saturation magnetizations for both are similar, the shapes of the hysteresis loops taken at $5 \mathrm{~K}$ are different. $\mathrm{Z}(0.5,8)$ exhibits a wasp-waist shape (Fig. 5(a)) while $Z(0,8)$ does not (latter not shown). We speculate that the origin of the ferromagnetic hysteresis is different for both samples. This will be discussed in the next section using element-specific analysis. 


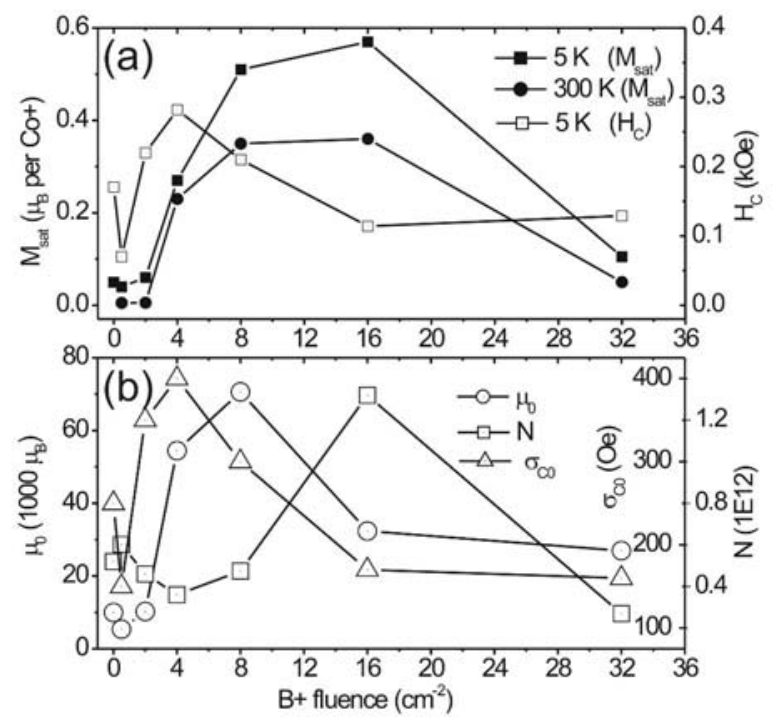

Fig. 6(a) Dependence of the saturation magnetization $\left(M_{\text {sat }}\right)$ and the coercive field $\left(\mathrm{H}_{\mathrm{C}}\right)$ on the boron fluence. (b) Dependence of the number of clusters $\mathrm{N}$ (per $\mathrm{cm}^{2}$ implanted), their moment $\mu_{0}$ and the dispersions $\sigma_{c 0}$ of $\mathrm{H}_{\mathrm{C}}$ at zero temperature and the $\mathrm{B}+$ fluence

In order to get further information about the areas with a large Co concentration in Fig. 3(b), M-H curves have been measured for all of the samples after both ZFC and FC at a field of 5000 Oe (not shown). No clear indication for a shift of the loops, i.e. no exchange bias effect hinting towards ferromagnetic/antiferromagnetic interfaces has been found. In addition, the $\mathrm{M}-\mathrm{H}$ curve, e.g. of sample $\mathrm{Z}(8,8)$, mounted perpendicular to the field was recorded at $5 \mathrm{~K}$ (Fig. 5(c)). It clearly represents the magnetically hard axis in contrast to embedded metallic crystalline Co clusters ${ }^{2}$.

\section{Discussion of the influence of boron on the magnetic properties}

For the ferromagnetic coupling of the Co moments several models should be considered

- $d-d$ double exchange ${ }^{17}$ 
- ferromagnetic superexchange ${ }^{17}$

- direct d-d overlap

The first two interactions involve ionic states of Co while the latter occurs in metallic Co, Co-Zn precipitates ${ }^{18}$ or other alloys involving metallic Co. For the interpretation of the magnetization data, we have to consider that $B$ tends to chemically reduce Co and to enhance formation of stable amorphous areas. On the other hand, the $\mathrm{ZnO}$ matrix becomes instable after ion beam impact and tends to locally decompose and thus to create Co-Zn-O complexes. Another origin of reduction of the saturation magnetization and magnetic softening of metallic Co precipitates (see Fig. 6) is the admixture of large amounts of B. This has been studied mainly for Fe-Co-B alloys ${ }^{19}$. Assuming the latter applies to the current system, the results shown in Fig. 6(a), suggest that there is an optimum B:Co ratio leading to a maximum $M_{\text {sat. }}$ In the case of the $Z(x, 8)$ samples, $x$ is in the range of $8 \ldots 16 \times 10^{16} \mathrm{~cm}^{-2}$ and $B: C o$ is in the range of 1...2. In order to test the validity of this explanation for a second set of samples, we implanted 0,8 and $32 \times 10^{16} \mathrm{~cm}^{-2}$ of $B$ and subsequently $16 \times 10^{16} \mathrm{Co}^{+} / \mathrm{cm}^{2}$ in Zno, i.e. the $\mathrm{B}$ :Co ratios are $0,0.5$ and 2 for these samples. The saturation magnetization at 5 $\mathrm{K}$ amounts to $0.35,0.6$ and $0.8 \mu_{\mathrm{B}} / \mathrm{Co}$ atom for the three different $\mathrm{B}$ fluences. Thus, the maximum has shifted towards larger fluencies but the B:Co ratio is in the range of 2 as for the samples $Z(x, 8)$.

To determine the electronic and magnetic states of the implanted Co, we performed $X A$ and $X M C D$ measurements at the Co $L_{2,3}$ edges of samples $Z(0,16), Z(8,16)$ and $Z(32,16)$, the series exhibiting largest values for $M_{\text {sat. }}$ The results are shown in Fig. 7 . The line shape of the Co XA spectrum for sample $Z(0,16)$ is indicative of a divalent ionic Co state (Fig. 7(a)), i.e. $\mathrm{Co}^{2+}$, as in $\mathrm{CoO}^{20-21}$. In contrast, the XMCD lineshape (Fig. 7 (b)) is very similar to that of Co metal. The magnitude of the XMCD signal 
corresponds to an average magnetic moment per Co atom that is about 15 times smaller than for Co metal. (The saturation magnetization of metallic crystalline Co amounts to $1.7 \mu_{\mathrm{B}}$ per Co atom.) Consequently, the XA signal arises from mainly paramagnetic $\mathrm{Co}^{2+}$ ions, as expected for $\mathrm{Co}$ doped Wurtzite $\mathrm{ZnO}$, and a small amount of metallic Co. In order to explain the rather large saturation magnetization of $Z(0,16)$ and the small deviations of the XMCD lineshape from that of metallic Co, we consider a second contribution to the ferromagnetic signal. This contribution can be related to ionic Co which develops saturation spin moment per ion of $3 \mu_{B}$ for $\mathrm{Co}^{2+}$ and $4 \mu_{\mathrm{B}}$ for $\mathrm{Co}^{3+}$. Ferromagnetically ordered Co ions have been found by Barla et al. for $\mathrm{Zn}_{0.75} \mathrm{Co}_{0.25} \mathrm{O}$ (Ref. 22) and Kobayashi et al. for $\mathrm{Zn}_{0.95} \mathrm{Co}_{0.05} \mathrm{O}$ (Ref. 23). Note that upon implantation of $\mathrm{ZnO}$ thin films on $\mathrm{Al}_{2} \mathrm{O}_{3}$ substrates with even larger $\mathrm{Co}^{+}$ fluencies, ferromagnetic Wurtzite $\mathrm{Zn}_{0.5} \mathrm{Co}_{0.5} \mathrm{O}$ is created ${ }^{24}$. Moreover, ferromagnetic defective $\mathrm{CoO}$ has been demonstrated ${ }^{25}$. In our case, a structurally disordered prestate of the sporadically occurring spinel $\mathrm{Zn}(\mathrm{Co}) \mathrm{Co}_{2} \mathrm{O}_{4}$ clusters might be the origin. Upon boron pre-implantation the XMCD lineshape does not change significantly but the XMCD signal becomes greatly enhanced, i.e. by about a factor of 10 , and the XA line shape becomes more metallic (Fig. $7(\mathrm{c})-(\mathrm{f}))$. In view of these results, we can explain the dependence of $\mathrm{M}_{\text {sat }}$ on the $\mathrm{B}^{+}$fluence shown in Fig. 6(a) in the following way: At low boron fluence, most of the Co ions are in ionic, paramagnetic state since there is still low reduction effect of the boron. $M_{\text {sat }}$ of $Z(0.5,8)$ therefore is comparable to that of $Z(0,8)$. With increasing boron fluence, the reduction becomes significant and the magnetization of the samples increases due to the growth of the size and number of the metallic clusters. The largest cluster density is reflected by the strongest interparticle interaction for $Z(4,8)$. The maximum magnetization per cluster is reached for $Z(8,8)$ as shown in Fig. $6(b)$. For further increase of fluence the number of clusters still increases but the moment per cluster decreases due to larger B 
content. This trend is shown for $Z(16,8)$ in Fig. $6(b)$. Further enhancement of the boron concentration renders the clusters more superparamagnetic due to magnetic softening and further reduction of $\mathrm{M}_{\text {sat. }}$. Admixture of $\mathrm{Zn}$ to those clusters is also likely and also results in lower values of $\mathrm{M}_{\text {sat }}$.
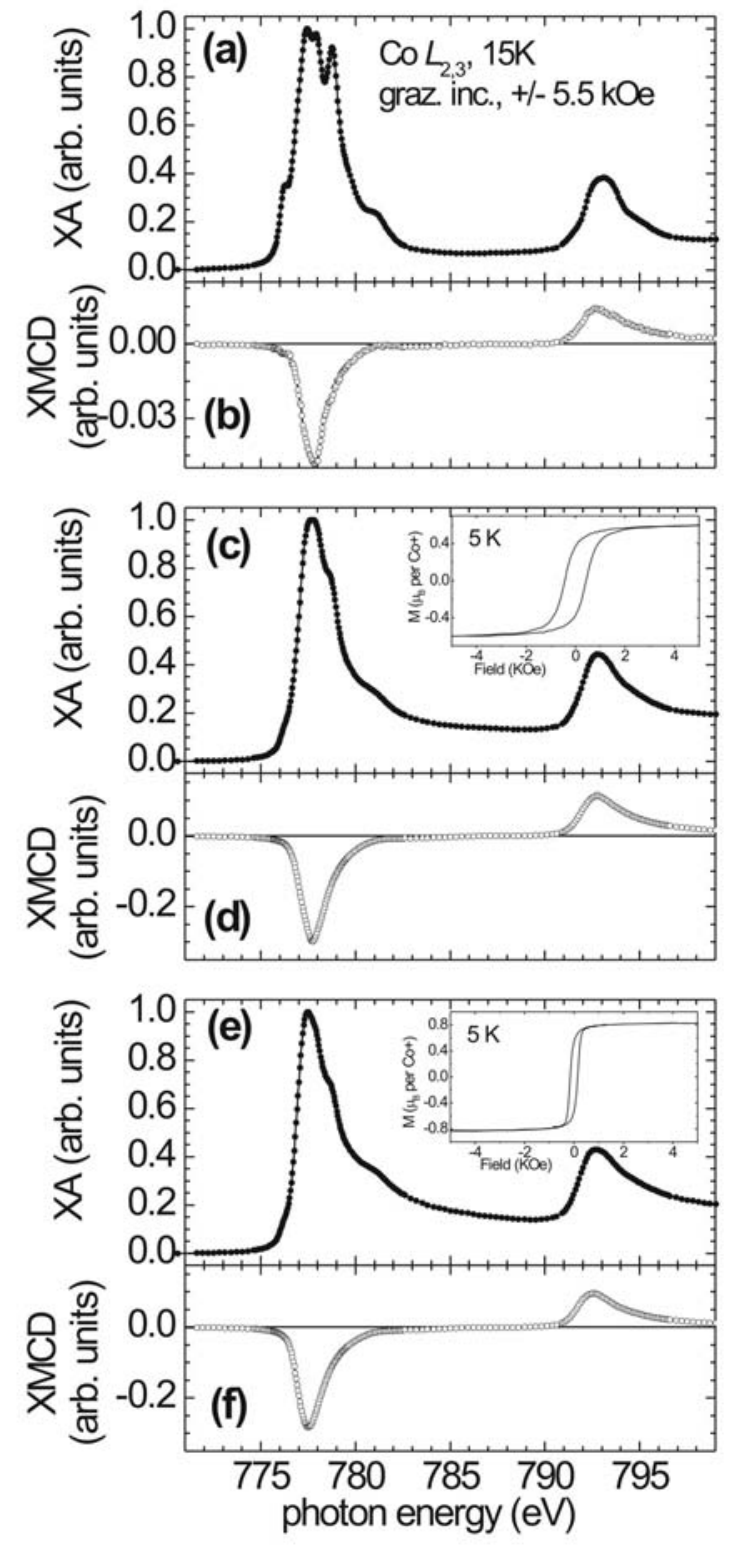

Fig. 7. (a), (c), (e) XA and (b), (d), (f) XMCD spectra at the $L_{2,3}$ absorption edges of Co. The magnetic field indicated was applied $60^{\circ}$ from the surface normal. Samples $Z(0,16)(a, b), Z(8,16)(c, d)$ and $Z(32,16)(e, f)$ have been measured. The labels of the 
axes are equal to (a). The insets in (c) and (e) display SQUID M-H measurements of the samples recorded at $5 \mathrm{~K}$.

\section{Electronic transport properties}

The transport properties of the samples are investigated by Hall measurements using the van der Pauw geometry. The virgin $\mathrm{ZnO}$ single crystals grown by hydrothermal method are semi-insulating with the carrier concentration of $10^{13} \mathrm{~cm}^{-3}$ and the mobility of $120 \mathrm{~cm}^{2} \mathrm{~V}^{-1} \mathrm{~s}^{-1}$ at room temperature. These parameters are comparable with those from other groups. Typically, the free charge carrier concentration amounts to $1 \times 10^{14} \mathrm{~cm}^{-3}$ (ref. 13,26 ) and the mobility to $200 \mathrm{~cm}^{2} \mathrm{~V}^{-1} \mathrm{~s}^{-1}$ (ref. 13). Note that the implantation leads to a relatively large charge carrier sheet concentration (range of $10^{15} \mathrm{~cm}^{-2}$ corresponding to $10^{20} \mathrm{~cm}^{-3}$ in a $75 \mathrm{~nm}$ thick film) and mobility (range of $10 \mathrm{~cm}^{2} \mathrm{~V}^{-1} \mathrm{~s}^{-1}$ ). These values are comparable to those achieved for $\mathrm{ZnO}$ based transparent conductive oxides (Ref. 28), while hydrothermal ZnO crystals are mostly insulating. Nevertheless, a dependence of the sheet resistance $R, n_{e}$, and $\mu$ on the boron fluence can be observed (Fig. 8). The trend of the sheet resistance $R$ and the charge carrier density $n_{e}$ with increasing $B^{+}$fluence (Fig. 8(a), (b)) nearly corresponds to that of the saturation magnetisation (Fig. 6). This suggests to associate the increase in saturation magnetisation with the increase of charge carrier density, e.g. from oxygen vacancy formation. It has been shown that oxygen vacancies do not provide shallow but rather deep donors ${ }^{25}$. Since the magnetic properties originate from amorphous metallic inclusions, the interpretation of the dependence of both $n_{e}$ and $\mu$ on the $B^{+}$fluence is rather difficult. The large $n_{e}$ and $\mu$ values are caused by defects from the Co implantation, while they are influenced in the range of one order of magnitude by the boron pre-implantation. Thus, a doping effect of boron plays no major role. Possible explanations of the dependence of $R, n_{e}$ 
and $\mu$ on the $\mathrm{B}^{+}$fluence could be related to the n-type doping effect of the boron. Indeed, the sheet resistivity of boron doped $\mathrm{ZnO}$ as a function of the boron concentration shows a minimum and $n_{e}$ shows a maximum ${ }^{4}$. The increase of $R$ starts at a boron concentration of $2 \times 10^{21} \mathrm{~cm}^{-3}$ which is in a similar range as our doping level for $Z(2,8)$. It has been explained with the creation of compensating $\operatorname{defects}^{4}$. The similarity between Figs. 6 and 8, i.e. corresponding extrema of $M_{\text {sat }}$ (maximum) and $\mathrm{n}_{\mathrm{e}}$ (maximum), indicates that the transport properties may also reflect the presence of low-resistivity (metallic) clusters at low Schottky barrier height with the surrounding host material keeping in mind that amorphous metals generally show larger resistivity as compared to crystalline systems. Similar to $M_{S}$, the initial decrease of the resistance $R$ and charge carrier concentration $n_{e}$ is related to the increasing reduction effect of the boron and thus the creation of metallic, low resistive clusters. The maximum of $n_{e}$ for $Z(8,8)$ reflects the maximum number of low resistive clusters. Further enhancement of the boron fluence lowers $N_{S}$ and enhances the sheet resistance. The enhancement of $R$ with increasing $B$ concentration in Fe-Co-B films is a well known phenomenon. It corresponds to the reduction of the saturation magnetization ${ }^{19}$. Thus, we can explain the correspondence between $M_{\text {sat }}, R$, and $n_{e}$. The origin of the sheet resistance minimum in Fig. 8(a) remains unidentified. Note that as in the case of the magnetic properties, the resistance $R$ of $Z(0,8)$ is significantly different from that of $Z(0.5,8)$ again indicating the different kinds of clusters present in these samples. 


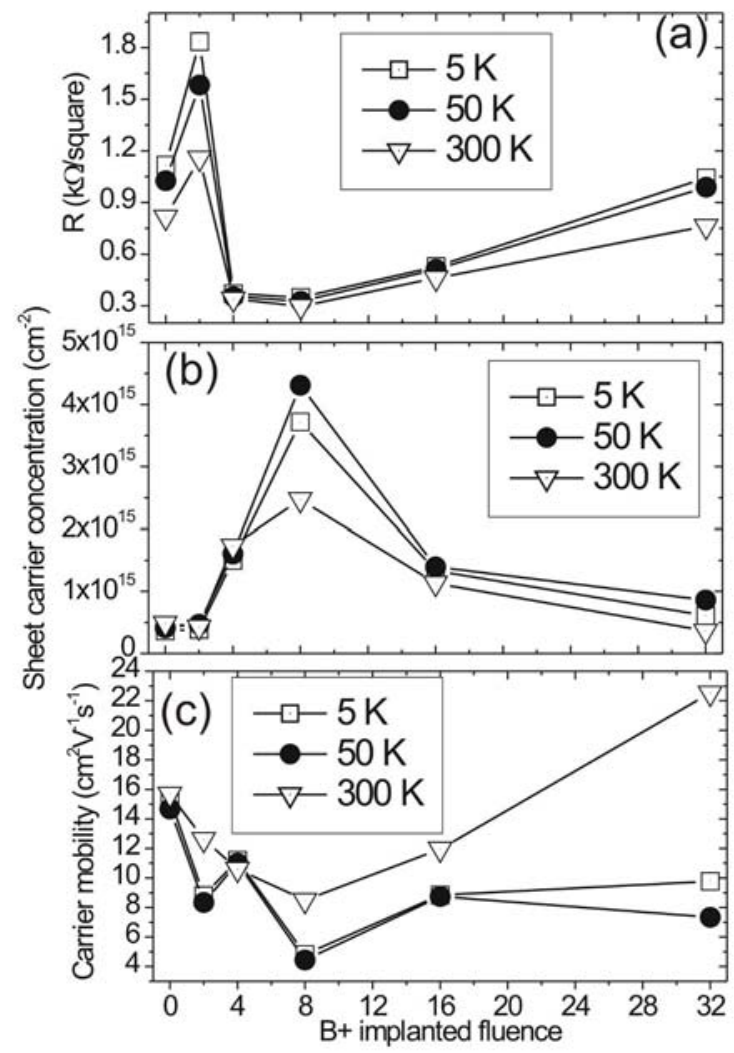

Fig. 8. Sheet resistance (a), sheet carrier concentration (b) and mobility (c) as a function of the implanted $\mathrm{B}^{+}$fluence measured at different temperatures (indicated). All samples have been implanted subsequently with $8 \times 10^{16} \mathrm{~cm}^{-2} \mathrm{Co}^{+}$.

The magneto-resistance (MR) measured in the van der Pauw geometry with the magnetic field perpendicular to the sample surface is shown in Fig. 9. For zero or low boron fluences, the MR (defined as $(R[H]-R[0]) / R[0] \times 100 \%)$ ) is positive. The observed positive MR is rather common for Co-doped $\mathrm{ZnO}$ and may be modelled by the action of the Lorentz force on the mobile charge carriers ${ }^{29}$ or by the quantum correction on the conductivity due to the s-d exchange interaction induced splitting of the conduction band ${ }^{30}$. The first explanation is most likely since a similar shaped positive MR effect could be measured also for one of the virgin samples (not shown). With increasing boron fluence, the negative MR becomes dominant at $5 \mathrm{~K}$. Small 
negative $\mathrm{MR}$ in Co-doped $\mathrm{ZnO}$ has been modelled by the field-induced suppression of weak localization ${ }^{30}$. Large negative MR in Ti-, $\mathrm{Cu}$-, and $\mathrm{Nd}$-doped $\mathrm{ZnO}$ has been modelled by considering the magnetic scattering of conduction electrons by the isolated magnetic ions ${ }^{31}$. In order to reveal a dependence on the normalized magnetization, we plot the MR as a function of the B fluence as shown in Fig. 9(d). The negative MR cannot be modelled by $\left(M / M_{S}\right)^{2}$ as known for granular giant MR materials $^{32}$. This proves that the boron pre-implanted, Co-implanted $\mathrm{ZnO}$ contains nonaligned ferromagnetic entities on a microscopic scale. We speculate, that the MR, especially at high fields, originates from disorder or paramagnetic defects introduced by the increasing $B^{+}$fluence. In contrast to $Z(0,16)^{10}$, anomalous Hall effect could not be detected for the $Z(x, 8)$ crystals, likely due to the low amount of closely located clusters in all samples. 

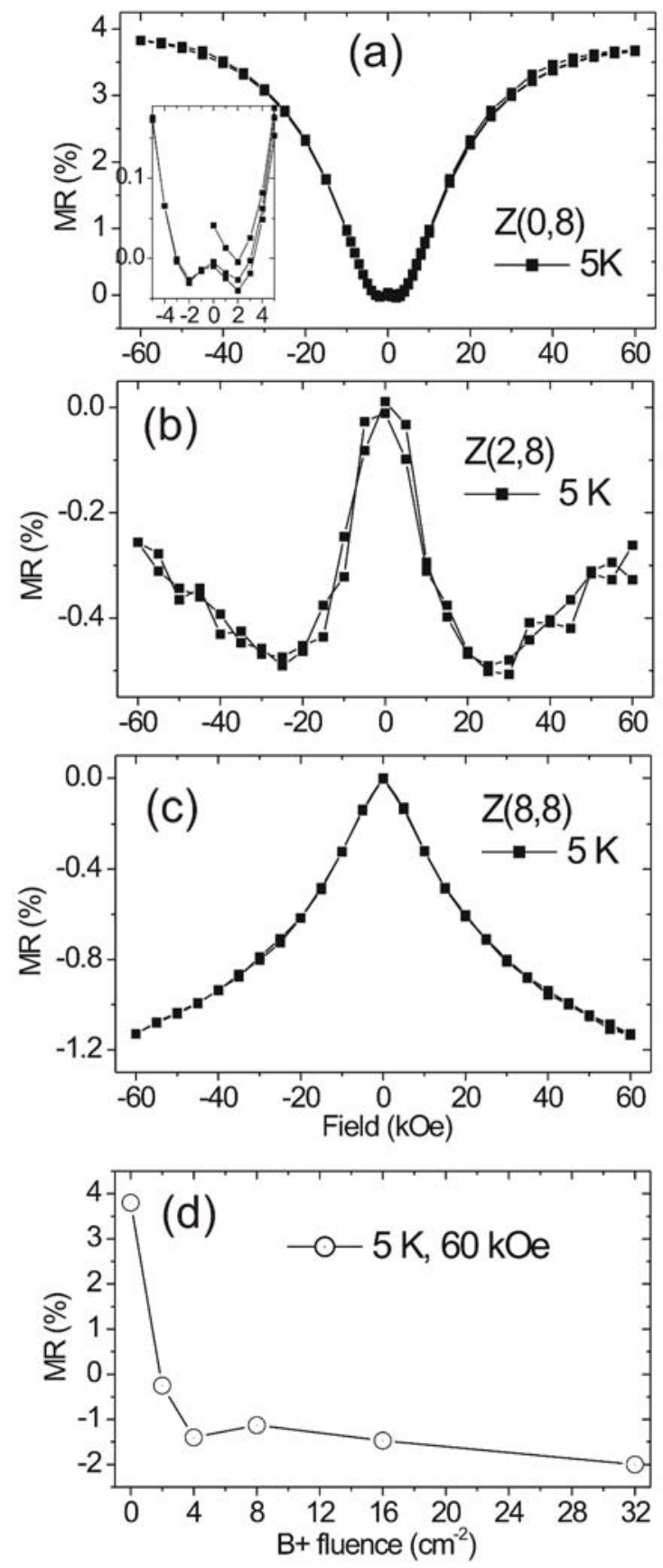

Fig. 9 (a), (b), (c). MR measurements for selected samples labelled according to Table I. (d) MR values at $5 \mathrm{~K}$ and $60 \mathrm{kOe}$ as a function of the $\mathrm{B}^{+}$fluence.

\section{Summary}

We studied the effect subsequently implanted of $\mathrm{B}$ and $\mathrm{Co}$ ions into $\mathrm{ZnO}(0001)$ single crystals on the magnetic and electronic properties of the system. Without boron pre- 
implantation, high fluence implanted Co appears dominantly in ionic state while the structure is dominated by the Wurtzite lattice of the $\mathrm{ZnO}$. It shows ferromagnetic order related predominantly to metallic Co. The presence of boron leads to two major effects:

1. Nearly total amorphisation of the implanted region which also contains crystalline clusters

2. Reduction of the implanted Co to a metallic state with ferromagnetic order The superparamagnetic/ferromagnetic phase cannot be identified by standard XRD scans. Since those observations are not induced by the implantation itself they are also expected to occur in deposited films. From a practical point of view, co doping of Co:ZnO with group III elements bears the danger of creating undetectable metallic ferromagnetic areas. Such doping is common especially for the enhancement of $n$ type conductivity or creation of oxygen vacancies.

The Advanced Light Source is supported by the Director, Office of Science, Office of Basic Energy Sciences, of the U.S. Department of Energy under Contract No. DEAC02-05CH11231. 
1. A. Ney, K. Ollefs, S. Ye, T. Kammermeier, V. Ney, T. C. Kaspar, S. A. Chambers, F. Wilhelm, and A. Rogalev, Phys. Rev. Lett. 100, 157201 (2008).

2. Shengqiang Zhou, K. Potzger, G. Talut, J. von Borany, W. Skorupa, M. Helm, and J. Fassbender, J. Appl. Phys. 103, 07 D530 (2008).

3. M. Gacic, G. Jakob, C. Herbort, H. Adrian, T. Tietze, S. Bruck, and E. Goering, Phys. Rev. B 75, 205206 (2007).

4. W. W. Wenas, A. Yamada, K. Takahashi, M. Yoshino, and M. Kongai, J. Appl. Phys. 70, 7119 (1991).

5. Z. Q. Chen, M. Maekawa, A. Kawasuso, S. Sakai, and H. Naramoto, J. Appl. Phys. 99, 093507 (2006).

6. Shengqiang Zhou, K. Potzger, H. Reuther, G. Talut, F. Eichhorn, J. von Borany, W. Skorupa, M. Helm, and J. Fassbender, J. Phys. D - Appl. Phys. 40, 964 (2007).

7. K. Potzger, Shengqiang Zhou, H. Reuther, K. Kuepper, G. Talut, M. Helm, J. Fassbender, and J. D. Denlinger, Appl. Phys. Lett. 91, 062107 (2007).

8. K. Potzger, S. Q. Zhou, H. Reuther, A. Mucklich, F. Eichhorn, N. Schell, W. Skorupa, M. Helm, J. Fassbender, T. Herrmannsdörfer, and T. P. Papageorgiou, Appl. Phys. Lett. 88, 052508 (2006).

9. K. Potzger, K. Küpper, Q. Xu, S. Zhou, H. Schmidt, M. Helm, and J. Fassbender, J. Appl. Phys. 104, 023510 (2008).

10. K. Potzger, Shengqiang Zhou, Qingyu Xu, A. Shalimov, R. Groetzschel, H. Schmidt, A. Mücklich, M. Helm, and J. Fassbender, Appl. Phys. Lett. 93, 232504 (2008).

11.S. O. Kucheyev, J. S. Williams, C. Jagadish, J. Zou, C. Evans, A. J. Nelson, and A.V. Hamza, Phys. Rev. B 67, 094115 (2003). 
12.W. Möller and W. Eckstein, Nucl. Instrum. Methods Phys. Res. B 2, 814 (1984).

13. Katsumi Maeda, Mitsuru Sato, Ikuo Niikura and Tsuguo Fukuda, Semicond. Sci. Technol. 20 (2005) S49-S54

14. T. M. Børseth, B. G. Svensson, and A. Yu. Kuznetsov, Phys. Scr. T126, 10 (2006).

15. S. Facsko, T. Bobek, A. Stahl, H. Kurz, T. Dekorsy, Phys. Rev. B 69, 153412 (2004).

16. T. Song, R. M. Roshko, and E. Dan Dahlberg, J. Phys.: Condens. Matter 13, 3443 (2001).

17. B. Belhadji, L. Bergqvist, R. Zeller, P. H. Dederichs, K. Sato, and H. Katayama-Yoshida, J. Phys. Condens. Matter 19, 436227 (2007).

18. T. C. Kaspar, T. Droubay, S. M. Heald, M. H. Engelhard, P. Nachimuthu, and S. A. Chambers, Phys. Rev. B 77, 201303(R) (2008).

19. Inyoung Kim, Jongryoul Kim, Ki Hyeon Kim, and Masahiro Yamaguchi, IEEE Trans. Magn. 40, 2706 (2004).

20. J.-Y. Kim, J.-H. Park, B.-G. Park, H.-J. Noh, S.-J. Oh, J. S.Yang, D.-H. Kim, S. D. Bu, T.-W. Noh, H.-J. Lin,H.-H. Hsieh, and C.T. Chen, Phys. Rev. Lett. 90, 017401 (2003).

21. T.J. Regan, H. Ohldag, C. Stamm, F. Nolting, J. Lüning, and J. Stöhr, R. L. White, Phys. Rev. B 64, 214422 (2001).

22. A. Barla, G. Schmerber, E. Beaurepaire, A. Dinia, H. Bieber, S. Colis, F. Scheurer, J.-P. Kappler, P. Imperia, F. Nolting, et al., Phys. Rev. B 76, 125201 (2007). 
23. M. Kobayashi, Y. Ishida, J. I. Hwang, T. Mizokawa, A. Fujimori, K. Mamiya, J. Okamoto, Y. Takeda, T. Okane, Y. Saitoh, Y. Muramatsu, A. Tanaka, H. Saeki, H. Tabata, and T. Kawai, Phys. Rev. B 72, 201201 (R) (2005).

24. N. Akdogan, A. Nefedov, K. Westerholt, H. Zabel, H.-W. Becker, C. Somsen, R. Khaibullin, and L. Tagirov, J. Phys. D. Appl. Phys. 41, 165001 (2008).

25. Dimple P. Dutta, Garima Sharma, P. K. Manna, A. K. Tyagi, and S. M. Yusuf, Nanotechnology 19, 245609 (2008).

26. G. H. Kassier, M. Hayes, F. D. Auret, M. Mamor, and K. Bouziane, J. Appl. Phys. 102, 014903 (2007).

27. A. Janotti and C. G.V. de Walle, Phys. Rev. B 76, 165202 (2007).

28. Cornelius, S.; Vinnichenko, M.; Shevchenko, N.; Rogozin, A.; Kolitsch, A.; Möller, W. submitted to Appl. Phys. Lett. (Hall effect mobility of $46 \mathrm{~cm}^{2} \mathrm{~V}^{-1} \mathrm{~s}^{-1}$, free electron density of $6 \times 10^{20} \mathrm{~cm}^{-3}$ )

29. P. Stamenov, M. Venkatesan, L. S. Dorneles, D. Maude, and J. M. D. Coey J. Appl. Phys. 99, $08 \mathrm{M} 124$ (2006).

30. Qingyu Xu, Lars Hartmann, Heidemarie Schmidt, Holger Hochmuth, Michael Lorenz, Daniel Spemann, and Marius Grundmann, Phys. Rev. B 76, 134417 (2007).

31. L. Hartmann, Q. Xu, H. Schmidt, H. Hochmuth, M. Lorenz, C. Sturm, C. Meinecke, and M. Grundmann, J. Phys. D 39, 4920 (2006).

32. John Q. Xiao, J. Samuel Jiang, and C. L. Chien, Phys. Rev. Lett. 68, 3749 (1992). 